\title{
Axial suspension test to assess pre-operative spinal flexibility in patients with adolescent idiopathic scoliosis
}

\author{
Philippe Büchler • Marcelo Elias de Oliveria • \\ Daniel Studer · Steffen Schumann · Guoyan Zheng • \\ Jacques Schneider $\cdot$ Carol C. Hasler
}

Received: 26 November 2013/Revised: 13 May 2014/ Accepted: 14 May 2014/Published online: 30 May 2014

(c) Springer-Verlag Berlin Heidelberg 2014

\begin{abstract}
Introduction An accurate description of the biomechanical behavior of the spine is crucial for the planning of scoliotic surgical correction as well as for the understanding of degenerative spine disorders. The current clinical assessments of spinal mechanics such as side-bending or fulcrum-bending tests rely on the displacement of the spine observed during motion of the patient. Since these tests focused solely on the spinal kinematics without considering mechanical loads, no quantification of the mechanical flexibility of the spine can be provided.

Methods A spinal suspension test (SST) has been developed to simultaneously monitor the force applied on the spine and the induced vertebral displacements. The system relies on cervical elevation of the patient and orthogonal radiographic images are used to measure the position of the vertebras. The system has been used to quantify the spinal flexibility on five AIS patients.

Results Based on the SST, the overall spinal flexibility varied between $0.3 \% / \mathrm{Nm}$ for the patient with the stiffer curve and $2 \% \mathrm{Nm}$ for the less rigid curve. A linear correlation was observed between the overall spinal flexibility and the change in Cobb angle. In addition, the segmental
\end{abstract}

P. Büchler $(\bowtie) \cdot$ M. E. de Oliveria · S. Schumann · G. Zheng Institute for Surgical Technology and Biomechanics,

University of Bern, Bern, Switzerland

e-mail: philippe.buechler@istb.unibe.ch

URL: http://www.istb.unibe.ch

D. Studer · C. C. Hasler

Orthopaedic Department, University Children's Hospital Basel,

Basel, Switzerland

J. Schneider

Radiology Department, University Children's Hospital Basel,

Basel, Switzerland flexibility calculated for five segments around the apex was $0.13 \pm 0.07 \% \mathrm{Nm}$, which is similar to intra-operative stiffness measurements previously published.

Conclusions In summary, the SST seems suitable to provide pre-operative information on the complex functional behavior and stiffness of spinal segments under physiological loading conditions. Such tools will become increasingly important in the future due to the everincreasing complexity of the surgical instrumentation and procedures.

Keywords Spine $\cdot$ Scoliosis $\cdot$ Pre-operative test · Stiffness · Flexibility

\section{Introduction}

Adolescent idiopathic scoliosis (AIS) represents the most frequent spinal deformity during growth and is of particular importance in view of the long life expectancy of those otherwise healthy individuals. This disease affects about $2 \%$ of adolescent females and is the main cause for spine surgery during growth [1]. The treatment strategy has not evolved much over the last decades, since it still includes rigid braces for moderate, $20^{\circ}-40^{\circ}$ curves and long bony fusions for significant curves.

In order to template a surgical intervention, a preoperative assessment of the spinal flexibility is critical. Flexibility describes the mathematical ratio between the three-dimensional (3D) displacement of the spine and the force vector that was used to generate this motion. However, the current clinical assessment of spinal stiffness is based on the vertebral displacements observed during motion of the patient. Most simply, the patient is advised to bend forward, backward or laterally [2,3], is bent manually 
without or with a fulcrum [4-9]. One of the major limitations of these techniques is that only the spinal kinematics is taken into account without quantifying the mechanical loads acting on the spine. In addition, these assessments are usually done in a single plane. As a result, no quantitative information on the functional flexibility of the degenerated segments can be obtained from these tests.

Different techniques have been proposed to standardize the force used in the pre-operative test such as suspension test $[10,11]$, push-traction films [12] and traction radiographs $[13,14]$. However, calculation of the mechanical stiffness of the spine remains limited by the complex mechanisms to transfer the forces applied on the patient to his vertebras. From a biomechanical point of view, the suspension tests are the most appealing. During elevation, the gravitational force is used to deform the spine with a load that can be estimated from the patient's weight. Even for suspension tests, it remains challenging to determine the load transferred to the vertebras. In the suspension test proposed by Lamarre et al. [11], the patient is lifted by a force applied under the patient's armpits. However, the motion of the shoulder joint relative to the spinal column affects the amount of load transferred to the spine. The cervical traction proposed by Ghista et al. [10] seems more appropriate to transmit the load directly to the patient's spine. However, the proposed system does not ensure a proper axial alignment of the load with the spine. In addition, none of the proposed techniques respects the 3D deformity of the spine, as they are all based on single radiographs.

The objective of this study was to develop and evaluate a new system for the pre-operative assessment of the flexibility of the patient's spine. The system should ensure a proper definition of the magnitude and direction of the load applied to the patient's spine and be able to evaluate the induced vertebral motion. The purpose of this study was to show the feasibility of this technique and to evaluate spinal flexibility on scoliotic patients.

\section{Material and methods}

Five patients suffering from AIS who were scheduled to undergo posterior instrumented spinal fusion were recruited in this study. The inclusion criteria were children $(15.4 \pm 1.81$ years old) with moderate to severe idiopathic spinal deformities. The present study was carried out in accordance with the ethical standards laid down in the 1964 Declaration of Helsinki for research involving human subjects. The investigation was approved by the local ethics committee of the UKBB, and both oral and written informed consent to participate in this study were obtained from patients and relatives after a full explanation of the study.
Axial traction was applied to the patients' spine using a spinal suspension test (SST), which consists of a frame structure supporting a traction platform (Fig. 1). In order to ensure that the load is always applied axially, the motor providing the traction force is mounted on a platform able to freely move in the horizontal plane. The SST is equipped with a motion controller driver (MCLM 3006, Faulhaber Minimotor SA, Switzerland); a DC-servomotor with integrated precision gearbox (2642W012CR, Faulhaber Minimotor SA, Switzerland). The patient's weight was constantly monitored during the experiment using a Nintendo Wii balance board (Nintendo, Kyoto, Japan). This setup allows a wireless recording of the weight and the balance of the patients during traction and has been shown to be accurate and suitable for clinical settings [15].

A standard cervical traction head halter has been used to ensure axial loading of the spine. The head halter was gently placed on the occiput and on the chin of the subjects. The head halter was attached to the traction system using a rope and provides a line of action for the load aligned with the spinal column. During the test, the subjects were standing on a rotating load platform, which allows the acquisition of antero-posterior (AP) and lateral (LA) radiographs using a conventional X-ray system (Arcoma Intuition, Växjö, Sweden). A tractive preload corresponding to five percent of the patient's body weight was applied for positioning the two degrees of freedom slider component in line with the patient's spinal axis. Antero-posterior and lateral radiographic images of the spine were acquired in the unloaded condition and after application of a traction force corresponding to $30 \%$ of the patient's body weight. The test was performed under quasi-static conditions to avoid viscoelastic effects and patient's discomfort. The medical staff also constantly monitored the patients during traction. The experimental data such as time stamp in milliseconds, forces distribution on the four Wii board sensors and the center of the resulting force was recorded at a frequency of $15 \mathrm{~Hz}$ and stored in a text file for further off-line processing.

The two orthogonal X-ray radiographs were used to reconstruct a 3D representation of the patient's spine. During acquisition of the images, a pelvic belt was rigidly attached to the patient's iliac crest. The belt included a custom-made calibration grid required to establish the correspondence between lateral and AP images as well as to estimate the projection parameters of the radiographic system used [16]. Cobb angles in the unloaded and loaded conditions were measured on the radiographic images as well as the segmental flexibility by measuring the disk angles. The data obtained with the spinal suspension test were also compared with conventional side-bending radiographs of the same patients. The Cobb end vertebras used to measure the Cobb angle were selected on the 
Fig. 1 The SST system has been designed to ensure an axis loading of the patients' spine. A platform allows rotating the patient to acquire orthogonal radiographic images before and after elevation. A Wii board is used to continuously measure the weight of the patient during the test
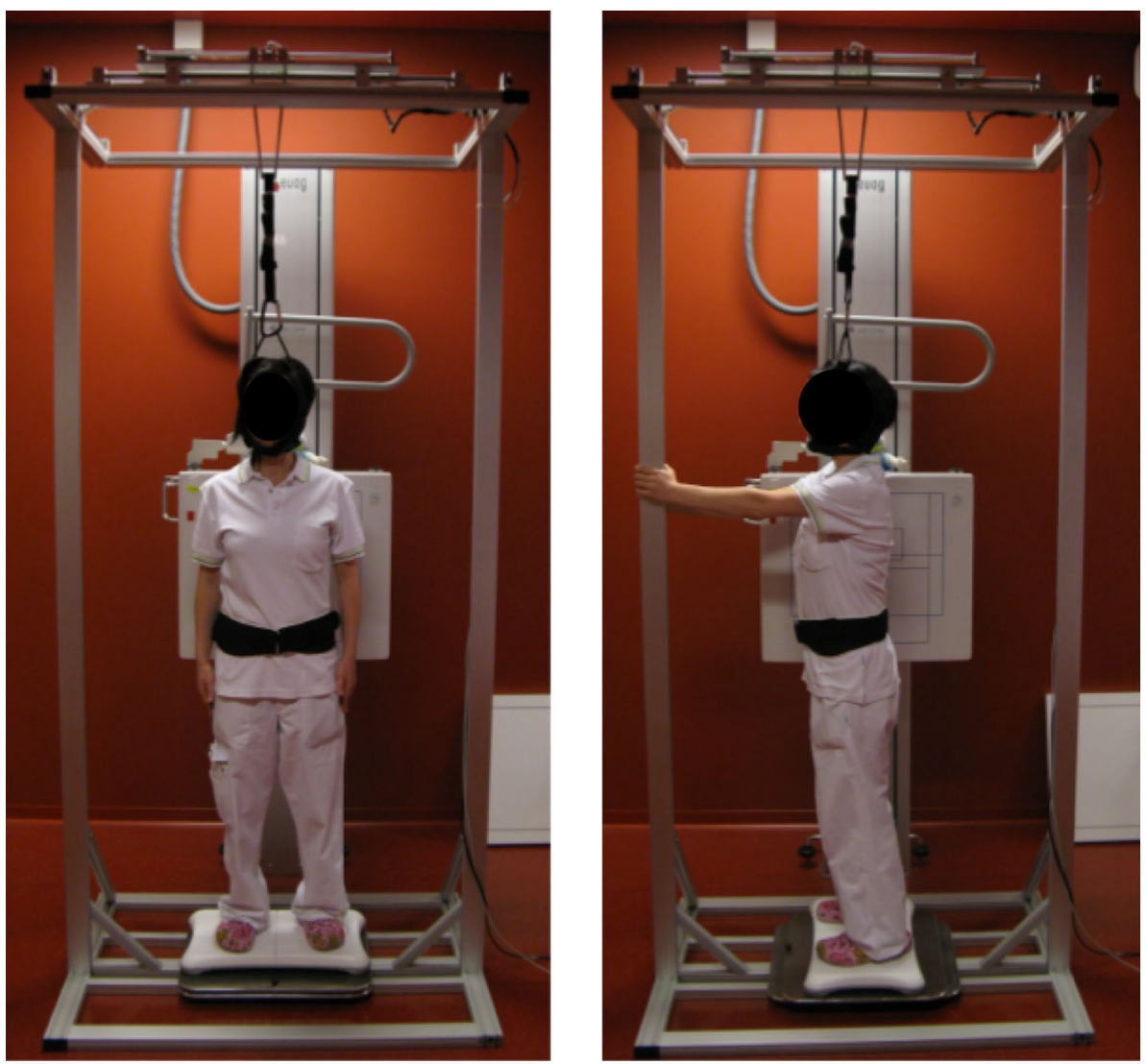

standing radiographs and were then kept anatomically constant to measure the Cobb angle on the images acquired after loading.

The flexibility of the spine was calculated based on the change of curvature induced by the loading force. The lever arm $\delta$ of traction force was defined as the distance between the apical vertebra and the direction of application of the load. The calibration system was used to determine the length of the lever arm in millimeters as well as its 3D orientation. The overall spinal flexibility was defined for each patient as the ratio between reduction of the curve and the loading torque:

$\zeta=\frac{\Delta_{\text {position }}}{F \delta}$

where the change in position $\Delta_{\text {position }}$ corresponds to the effect of loading on the Cobb angle, $F$ is the force applied on the patients' spine and $\delta$ is the distance between the apex vertebra and the vertical axis.

The segmental flexibility was also calculated for the disk at the apex of the scoliotic curve as well as for the two disks above and below the apex. The technique presented by Hasler et al. [17] has been used to measure the disk angles. The disk angles were measured before and after traction as straight lines along the inferior endplate of the upper and the superior endplate of the lower vertebra in a segment. Similar to the overall flexibility, the coronal segmental flexibility was calculated for each segment as the ratio between and changes in the disk angles and the traction moment acting on the vertebras.

\section{Results}

With the spinal suspension test, axial traction has been used to evaluate pre-operative overall and segmental stiffness of the curves of five patients with AIS (Fig. 2). The Cobb angle of the major curve was $<60^{\circ}$ in two patients, and $>60^{\circ}$ in three patients. A traction load of $30 \%$ of total body weight could be used without causing significant discomfort or pain in all patients. By using the SST, the Cobb angle of the major curves could be reduced between $5^{\circ}$ and $28^{\circ}$, whereas a reduction between $16^{\circ}$ and $45^{\circ}$ were achieved in the side-bending radiographs (Table 1). Also average curve reduction was higher for the side-bending radiographs $\left(30^{\circ} \pm 13^{\circ}\right)$ compared to axial traction radiographs $\left(12^{\circ} \pm 9^{\circ}\right)$. No correlation was found between the reduction of the curve obtained with the sidebending technique and the SST (Spearman coefficient $\rho=0.2$ ). Within the patients included in this study, no correlation was found between the reduction of the curve induced by the SST and the initial Cobb angle. 

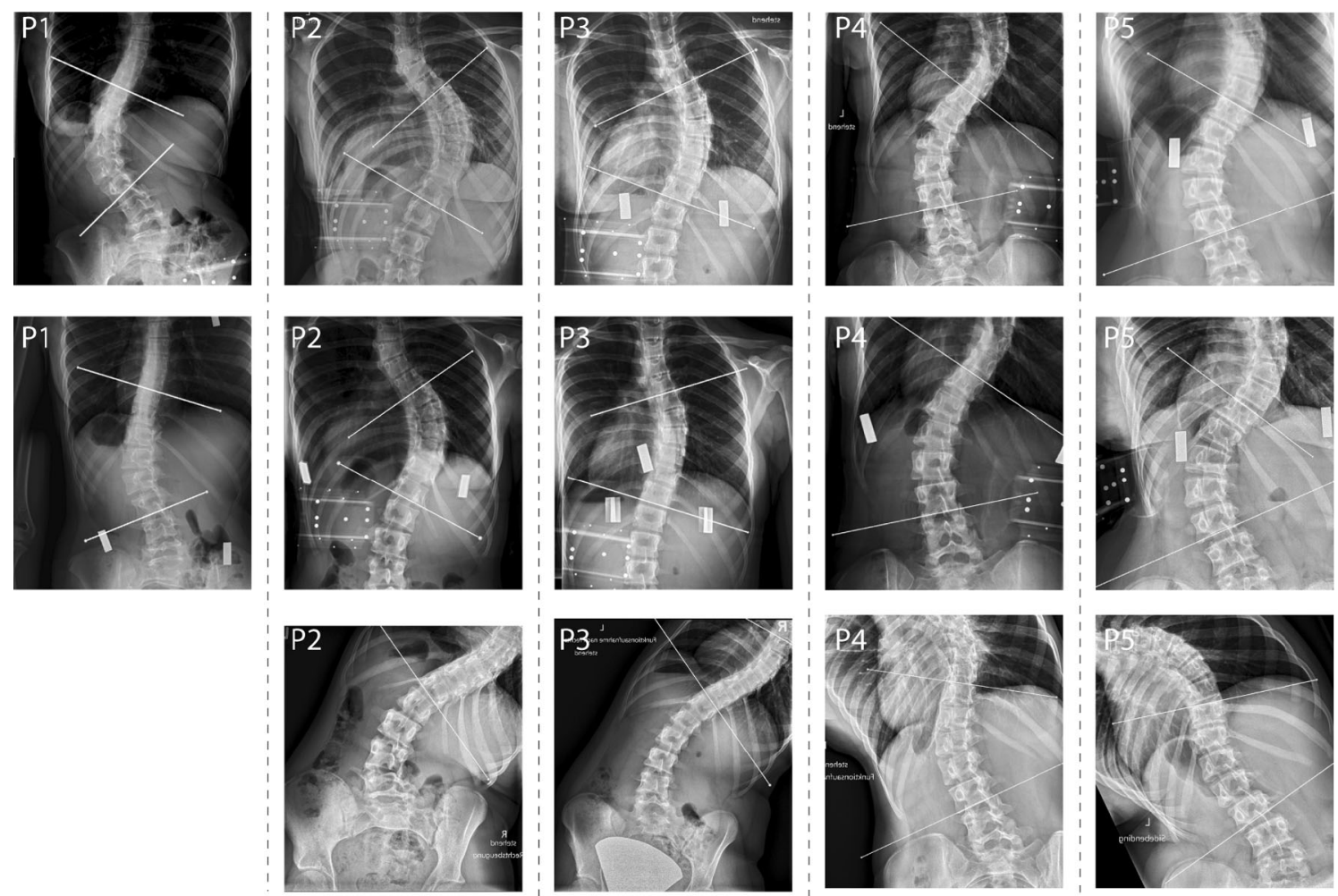

Fig. 2 Pre-operative radiographs obtained for the five patients. The first row corresponds to the antero-posterior radiographs in normal standing position, the second row to the SST test (axial quasi-statistic load corresponding to $30 \%$ of the patient's body weight) and the third row corresponds to the lateral side-bending test
Table 1 Patient information and Cobb angle measured in standing position, side bending and after elevation

\begin{tabular}{|c|c|c|c|c|c|c|c|c|}
\hline \multirow[t]{2}{*}{ Patient \# } & \multirow{2}{*}{$\begin{array}{l}\text { Age } \\
\text { (years) }\end{array}$} & \multirow[t]{2}{*}{ Apex } & \multirow{2}{*}{$\begin{array}{l}\text { Weight } \\
(\mathrm{kg})\end{array}$} & \multirow{2}{*}{$\begin{array}{l}\text { BMI } \\
\left(\mathrm{N} / \mathrm{m}^{2}\right)\end{array}$} & \multirow{2}{*}{$\begin{array}{l}\text { Load } \\
(\mathrm{N})\end{array}$} & \multicolumn{3}{|c|}{ Cobb angle $\left(^{\circ}\right)$} \\
\hline & & & & & & Standing & Bending & Suspension \\
\hline 1 & 15 & L1 & 58 & 20 & 175 & 67 & - & 39 \\
\hline 2 & 16 & T9 & 55 & 20 & 166 & 71 & 26 & 63 \\
\hline 3 & 13 & $\mathrm{~T} 8$ & 53 & 21 & 158 & 46 & 26 & 34 \\
\hline 4 & 18 & $\mathrm{~T} 8$ & 47 & 18 & 142 & 50 & 33 & 46 \\
\hline 5 & 15 & L1 & 56 & 20 & 168 & 60 & 23 & 50 \\
\hline
\end{tabular}

The patients included in this study had almost similar total body weights; therefore, comparable traction forces could be applied. The average traction load was $162 \pm 12 \mathrm{~N}$ (between 142 and $175 \mathrm{~N}$ ). Nevertheless, the spinal flexibility calculated using the SST showed large variations between the patients. The most stiff curve had a flexibility of $0.3 \% / \mathrm{Nm}$, which is six times less than the less rigid curve that had a stiffness of $2 \circ / \mathrm{Nm}$. An linear relationship can be observed between spinal flexibility and the correctability of the curve expressed as the change in Cobb angle before and after traction; stiff curves showed little changes in Cobb angle under traction, while flexible curves showed large changes in Cobb angle after applying traction (Fig. 3). On the other hand, no correlation was found between the change in Cobb angle measured on side- bending radiographs and the spinal stiffness obtained using the SST.

Segmental flexibility was calculated for the five segments around the apex of the major curve (Fig. 4). The flexibility of the curve was lower at the apex and increased with increasing distance from the apex. The average segmental flexibility calculated for the traction radiographs was $0.13 \pm 0.07 \%$ $\mathrm{Nm}$, however larger variations were observed between the patients (flexibility between 0.07 and $0.20 \% \mathrm{Nm}$ ).

\section{Discussion}

A standardized test setup has been proposed to assess overall as well as segmental spinal flexibility in patients 


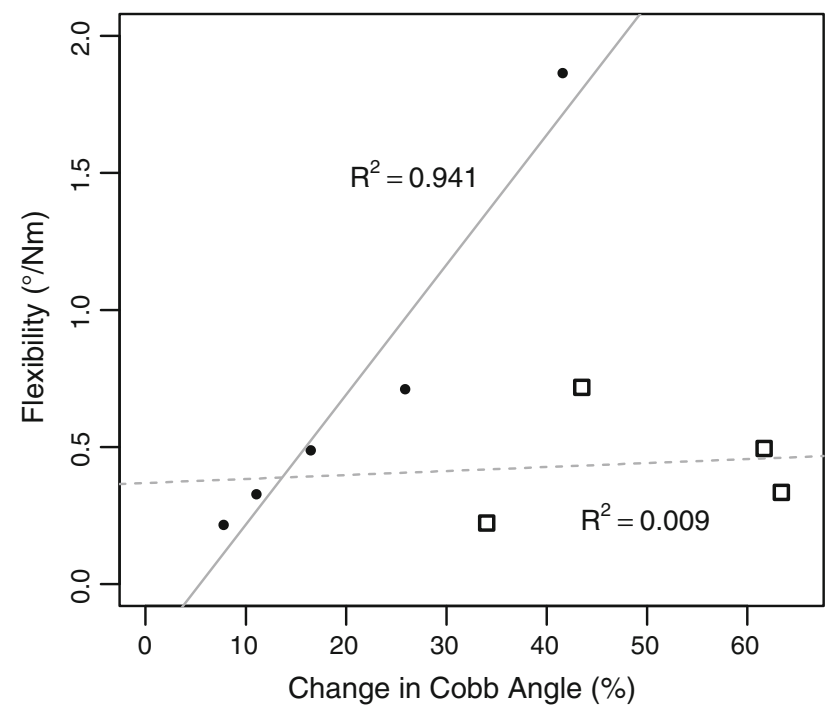

Fig. 3 Overall flexibility of the curve as a function of the change in Cobb angle for the SST (solid dots) and for the side bending (squares). The change in Cobb angle was measured on the frontal radiographs of the SST and side-bending tests, respectively. Since the flexility cannot be calculated based on the side-bending test, the value obtained with the SST was used for both datasets. A linear relation was found for the SST, while no correlation was observed in the case of the side-bending test

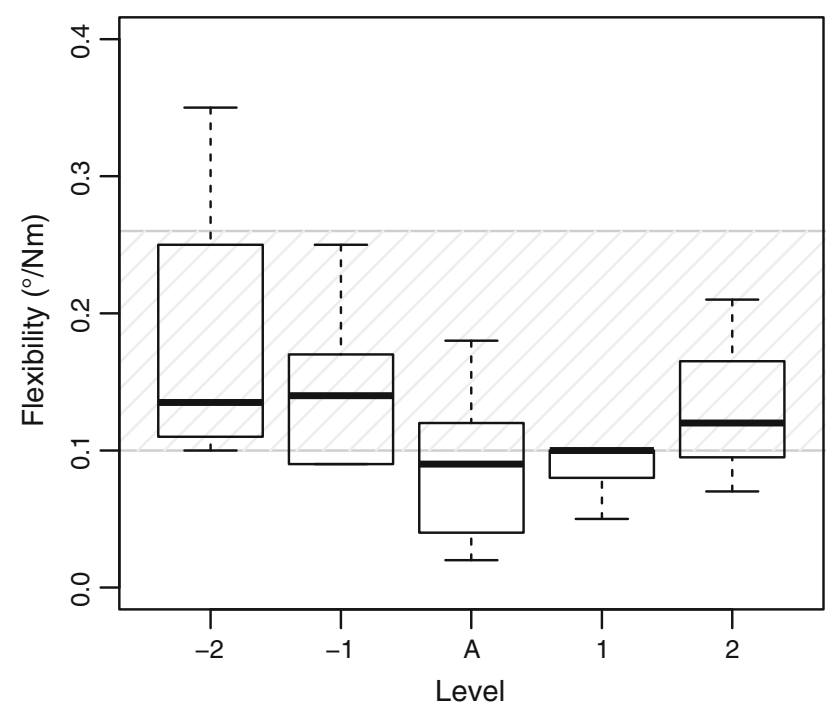

Fig. 4 Segmental flexibility calculated for five segments around the apex of the curve. The flexibility measured with the SST was lower at the apex and increased with the distance from the apex. The shaded region corresponds to the flexibility measured intra-operatively on eight motion segments around the apex of two patients (average \pm standard deviation) using a custom-made distraction forceps [18]

with AIS in a reproducible manner. The amount of load is continuously monitored during elevation of the patients. Correction of the spinal deformity is achieved based on the patients' own weight. Two perpendicular X-ray acquisitions enable a 3D reconstruction of the patients' spines.

All five patients who were included in this pilot study showed a reduction of the Cobb angle of their major curve, although the degree of correction under traction is less compared to side-bending radiographs. In this study, the load that was applied was limited to $30 \%$ of the patient's body weight. Higher loads could be safely applied with the SST, which would likely lead to larger corrections. However, it remains unclear if a large displacement of the vertebras during the clinical test is required to provide reliable information on spinal biomechanics. Results also indicated that the amount of correction obtained in this study was sufficient to accurately measure vertebral displacements on the radiographic images and to assess the mechanical stiffness of the spines. The lever arm of the elevation force has been used to calculate the spinal stiffness. The torque applied to the spine is directly related to the severity of the deformity, resulting in higher torques with more severe deformities. Therefore, the SST is likely to be more effective in correcting larger curves compared to smaller ones.

The values for the overall flexibility obtained in the present study are lower than the results published by Lamarre et al. [11], who were also using suspension-based technique. The lower values can be explained by the different approach used to apply the load on the spine; shoulder motion during the elevation make the estimation of the force acting on the vertebra difficult when the load is applied on the armpits of the patients. The cervical traction use in the present study allows a more direct loading of the spine. In addition, we used a calibration system to ensure an accurate measurement of the distance on the radiographs, as well as bi-planar images to measure the lever arm in three dimensions.

The segmental flexibility calculated pre-operatively can be compared with intra-operative measurements. Reutlinger et al. [18] measured the flexibility of eight motion segments on two patients using a custom-made distraction forceps. The average flexibility on the convex side was $0.18 \pm 0.08^{\circ} / \mathrm{Nm}$, which is similar to the values obtained pre-operatively with the SST $(0.14 \pm 0.09 \% / \mathrm{Nm})$. The lower flexibility reported with the present system can be explained by the muscular activation during the suspension test, while muscles were fully relaxed during the intraoperative measurements. The moment applied during the traction test was also higher than the $5 \mathrm{Nm}$ used intraoperatively. The non-linear moment-angle relationship of the soft tissue could also explain the decreased flexibility that was observed.

The results obtained with the SST differ from the sidebending radiographs. Although side bending induces more deformation to the tissues, a measure of the force acting on 
the spine to provide quantitative information about its stiffness is missing. Our results showed a strong relationship between the changes and spinal flexibility. They also demonstrate that mechanical side-bending information cannot be compared with the flexibility information retrieved from our suspension test. It appears that both examinations address different mechanical properties, and that only the suspension test is able to provide a quantitative flexibility.

The mechanical description used in this study is a strong simplification of the complex spinal biomechanics. Flexibility was used to quantify spinal mechanics, which measures the overall behavior of the structure (i.e., how much it deforms under a given load) and not the intrinsic mechanical properties of the tissues. The measured flexibility results from the combined effect of the muscles, ligaments, annulus, disk and other internal structures. It is currently not possible to determine the relative contribution of each of these structures to the overall flexibility. This is a limitation of this study, which does not differ on this aspect from traditional clinical assessment techniques. In addition, different level of muscle contraction will lead to different overall stiffness for the same patient. For this reason, the patients were instructed to relax during the test. Since the patients did not report pain during the evaluation, it is reasonable to believe that a good level of relaxation was possible. However, the problem of muscle contraction during flexibility testing is not new. Similar problem occurs during current clinical tests such as fulcrum bending. The problem is even more obvious for side bending, where the patients are asked to actively change the shape of their spine.

Another limitation of the suspension test concerns the traction load, which is not directly applied to the patient thoracic spine, but transmitted by the flexible occipitocervical junction. However, the flexibility is measured when the system is in static equilibrium. The eventual motions of the cervical vertebras during the loading phase will not affect the amount and direction of the force applied to the thoracic section of the spine, as long as the point of application of the traction load lies close to the spinal axis. The head halter ensures this alignment by application of the vertical load at the level of the ears of the patients. Further validation to quantify the accuracy of the measurement technique would require intra-operative stiffness measurement on the same patients that underwent suspension tests. This validation phase requires extensive work to provide accurate intra-operative stiffness measurement, which is outside the scope of the present study.

The 3D information on the patient's scoliotic curve is obtained from the bi-planar radiographic images. This information is obtained using conventional radiographic systems and has been used to calculate the lever arm of the force that was applied to the spine. Even though 3D imaging modalities such as computed tomography (CT) or magnetic resonance imaging (MRI) could be used to generate more accurate $3 \mathrm{D}$ models of the spine, only static information in non-weight-bearing supine position would be available. An alternative approach relies on the acquisition of EOS images (EOS imaging SA, Paris, France). This system is based on two orthogonal projections acquired at the same time using a low-dose radiation system and has been shown to be suitable to reconstruct the spine in 3D [19-21]. This system could also be used to quantify the vertebral motion induced by the elevation test. Though accurate and having potential for further exploration, the EOS is currently restricted due to its high acquisition and maintenance costs as well as the time required to generate the $3 \mathrm{D}$ reconstruction.

Unlike conventional systems that estimate spinal mechanics based on the vertebral kinematic without considering force information, the proposed pre-operative test enables a quantification of spinal stiffness. In addition, the segmental flexibility can be quantified for several segments along the curve. The data obtained on five patients favorably match with data collected intra-operatively. A precise evaluation of the patients' stiffness is important, since it is a prerequisite for targeted and individualized therapeutic conservative and operative strategies. This opens the door for patient-specific planning and optimization of surgical interventions [22]. Inclusion of stiffness data in patientspecific finite element models of their spines would not only provide a functional, dynamic planning tool but also serve as a base for the further development of novel nonfusion strategies for the correction of spinal deformities during growth.

Acknowledgments The work is supported by the Swiss National Science Foundation (SNSF) via the project 320030_138527.

Conflict of interest None.

\section{References}

1. Weinstein SL, Dolan LA, Cheng JCY et al (2008) Adolescent idiopathic scoliosis. Lancet 371:1527-1537. doi:10.1016/S01406736(08)60658-3

2. Hamzaoglu A, Talu U, Tezer M et al (2005) Assessment of curve flexibility in adolescent idiopathic scoliosis. Spine (Phila Pa 1976) 30:1637-1642

3. Hayes MA, Howard TC, Gruel CR, Kopta JA (1989) Roentgenographic evaluation of lumbar spine flexion-extension in asymptomatic individuals. Spine (Phila Pa 1976) 14:327-331

4. Cheung KMC, Lu DS, Zhang H, Luk KDK (2006) In-vivo demonstration of the effectiveness of thoracoscopic anterior release using the fulcrum-bending radiograph: a report of five cases. Eur Spine J 15(Suppl 5):578-582. doi:10.1007/s00586005-0027-2 
5. Cheung KMC, Luk KD (1997) Prediction of correction of scoliosis with use of the fulcrum bending radiograph. J Bone Joint Surg Am 79(8):1144-1150

6. Hay D, Izatt MT, Adam CJ et al (2008) The use of fulcrum bending radiographs in anterior thoracic scoliosis correction: a consecutive series of 90 patients. Spine (Phila Pa 1976) 33:999-1005. doi:10.1097/BRS.0b013e31816c8343

7. Klepps SJ, Lenke LG, Bridwell KH et al (2001) Prospective comparison of flexibility radiographs in adolescent idiopathic scoliosis. Spine (Phila Pa 1976) 26:E74-E79

8. Luk KDK, Lu DS, Cheung KMC, Wong YW (2004) A prospective comparison of the coronal deformity correction in thoracic scoliosis using four different instrumentations and the fulcrum-bending radiograph. Spine (Phila Pa 1976) 29:560-563

9. Hempfing A, Ferraris L, Koller $\mathrm{H}$ et al (2007) Is anterior release effective to increase flexibility in idiopathic thoracic scoliosis? Assessment by traction films. Eur Spine J 16:515-520

10. Ghista DN, Viviani GR, Subbaraj K et al (1988) Biomechanical basis of optimal scoliosis surgical correction. J Biomech 21:77-88

11. Lamarre M-E, Parent S, Labelle H et al (2009) Assessment of spinal flexibility in adolescent idiopathic scoliosis: suspension versus side-bending radiography. Spine (Phila $\mathrm{Pa}$ 1976) 34:591-597. doi:10.1097/BRS.0b013e318193a23d

12. Chen Z-Q, Wang C-F, Bai Y-S et al (2011) Using precisely controlled bidirectional orthopedic forces to assess flexibility in adolescent idiopathic scoliosis: comparisons between push-traction film, supine side bending, suspension, and fulcrum bending film. Spine (Phila Pa 1976) 36:1679-1684. doi:10.1097/BRS. 0b013e31820e6265

13. Hamzaoglu A, Talu U, Tezer M et al (2005) Assessment of curve flexibility in adolescent idiopathic scoliosis. Spine (Phila Pa 1976) 30:1637-1642

14. Takahashi S, Passuti N, Delécrin J (1997) Interpretation and utility of traction radiography in scoliosis surgery. Analysis of patients treated with Cotrel-Dubousset instrumentation. Spine (Phila Pa 1976) 22:2542-2546
15. Clark RA, Bryant AL, Pua Y et al (2010) Validity and reliability of the Nintendo Wii Balance Board for assessment of standing balance. Gait Posture 31:307-310. doi:10.1016/j.gaitpost.2009. 11.012

16. Schumann S, Dong X, Puls M, et al. (2012) Calibration of C-arm for orthopedic interventions via statistical model-based distortion correction and robust phantom detection. In: 9th IEEE international symposium biomedical imaging. IEEE, pp 1204-1207

17. Hasler C-C, Hefti F, Büchler P (2010) Coronal plane segmental flexibility in thoracic adolescent idiopathic scoliosis assessed by fulcrum-bending radiographs. Eur Spine J 19:732-738. doi:10. 1007/s00586-010-1320-2

18. Reutlinger C, Hasler C, Scheffler K, Büchler P (2012) Intraoperative determination of the load-displacement behavior of scoliotic spinal motion segments: preliminary clinical results. Eur Spine J. doi:10.1007/s00586-012-2164-8

19. Illés T, Tunyogi-Csapó M, Somoskeöy S (2010) Breakthrough in three-dimensional scoliosis diagnosis: significance of horizontal plane view and vertebra vectors. Eur spine J 20:135-143. doi:10. 1007/s00586-010-1566-8

20. Ilharreborde B, Sebag G, Skalli W, Mazda K (2013) Adolescent idiopathic scoliosis treated with posteromedial translation: radiologic evaluation with a 3D low-dose system. Eur Spine J. doi:10.1007/s00586-013-2776-7

21. Ilharreborde B, Steffen JS, Nectoux E et al (2011) Angle measurement reproducibility using EOS three-dimensional reconstructions in adolescent idiopathic scoliosis treated by posterior instrumentation. Spine (Phila Pa 1976) 36:E1306-E1313. doi:10. 1097/BRS.0b013e3182293548

22. Elias de Oliveira M, Hasler C-C, Zheng G et al (2011) A multicriteria decision support for optimal instrumentation in scoliosis spine surgery. Struct Multidiscip Optim 45:917-929. doi:10. 1007/s00158-011-0732-x 